\title{
Prospective Assessment of Catheter Migration in Implanted Vascular Access Devices for Adjuvant Colorectal Cancer Chemotherapy
}

\author{
Pia Osterlund ${ }^{1}$, Päivi Valta ${ }^{2}$, Sören Bondedstam ${ }^{3}$, Leena Lindgren ${ }^{4}$ \\ ${ }^{1}$ Department of Oncology, Helsinki University Central Hospital and University of Helsinki, Helsinki, Finland; ${ }^{2}$ Department of \\ Anaesthesiology, Helsinki University Central Hospital, Jorvi Hospital, Espoo, Finland; ${ }^{3}$ Department of Radiology, Helsinki \\ University Central Hospital, Helsinki, Finland; ${ }^{4}$ Department of Anaesthesia, Tampere University Hospital and University of Tampere, \\ Tampere, Finland. \\ Email: pia.osterlund@hus.fi
}

Received October $1^{\text {st }}, 2012$; revised October $31^{\text {st }}, 2012$; accepted November $11^{\text {th }}, 2012$

\begin{abstract}
Background: Chemotherapy in colorectal cancer is usually administered as continuous infusion of 5-fluorouracil, often in combination with oxaliplatin or irinotecan. Targeted drugs are most efficient and tolerable in conjunction with continuous infusion dosing. Implanted venous access devices (VAD) are the prerequisite for continuous infusion administration. The reported catheter migration frequency with VAD is $0 \%-3.5 \%$. The purpose of this case-control study was to evaluate the predisposing factors of catheter migration. Methods: We inserted VADs in 88 radically operated colorectal cancer patients randomized to adjuvant 48-hour-infusion chemotherapy repeated every 14 days, altogether 12 times over 24 weeks. Three out of 88 patients (3.4\%) had a symptomatic catheter migration from the superior caval vein into the internal jugular vein. The fourth case had chemotherapy for osteosarcoma. These 4 cases were compared with 12 controls from the same 88 patient study population, matched for age, sex, body mass index (BMI), physical activity level and right subclavian insertion site. Tip position, port model, complications, catheter length and material was studied. The post insertion catheter tip position in the chest X-ray was numbered from 1 (in subclavia) to 8 (in atrium). Results: The four cases, all male, had a median position of 3 (range 3 - 4) and controls 6 (range 4 - 8), P = 0.004, median difference $3\left(\mathrm{CI}_{95 \%} 1-5\right)$. At notification of migration the patients had experienced discomfort in the neck region starting 5 to 15 days before at strenuous upper extremities activity with Valsalva maneuvers. Conclusion: Optimal catheter tip position when sitting is in the right atrium or low in the superior vena cava to avoid migration. Patients with VADs should avoid strenuous activity with Valsalva maneuvers.
\end{abstract}

Keywords: Central Venous Catheterization; Catheter Migration; Complication; Colorectal Cancer; Vascular Access Device

\section{Introduction}

Colorectal cancer is the third most common cancer cancer in the Western world with 1,000,000 new cases a year [1]. Chemotherapy with 5-fluorouracil (5-FU) is the cornerstone in both the adjuvant and metastatic settings. Combination chemotherapy with 5-FU and oxaliplatin or irinotecan is better tolerated as continuous infusion administration than perorally or as intravenous boluses $[2,3]$. Implanted vascular access devices (VAD) are the most convenient and safe administration route for these cytotoxics [4]. With biological treatments the importance of the dosing schedule in combination chemotherapy has become even more important as continuous infusion based seem most efficient [5-9].

Implanted VADs cause immediate complications at insertion such as bleeding, pneumo- and hemothorax. The most typical long-term complications are thrombosis and infection, but rarer complications are skin damage, vascular erosion, catheter pinch-off and migration [10]. The frequency of catheter tip migration, even after adequate initial positioning, to an undesirable position is seen in $0 \%-3.5 \%$ of cases [11-15]. However, the definition of catheter migration in these series varies and the percentage of cases with full reversal of catheter to an unintended vein is unknown. No prospective studies with regular catheter check-ups have been performed to our knowledge. In one study the subclavian tip positioning, compared to atrial tip positioning, was thought to be a predisposing factor for migration [16]. In Hickman lines (tunneled central catheters) catheter migration or dis- 
lodgement was noted in 7\% of cases [17]. When all complications are considered, totally implanted ports seem to have less complications than exteriorized catheters $(31 \%$ vs. $58 \%)[16,18]$.

We performed a prospective randomized study in 177 radically operated colorectal cancer patients receiving adjuvant chemotherapy during six months, either as repeated peripheral injections (89 patients) or via totally implanted VADs for continuous 48-hour infusions in home care (88 patients). 5-fluorouracil in combination with leucovorin was administered according to the Mayo regimen or the modified LV5FU2 dosing regimen [19]. Tolerability was the primary endpoint of this study and special emphasis was prospectively put on VAD safety. Three out of 88 (3.4\%) VAD patients had a symptomatic catheter migration from the superior vena cava cranially into the internal jugular vein. An additional patient with catheter migration during chemotherapy was also noted.

The aim of this study was to present four cases of catheter migration and to evaluate the predisposing factors of catheter migration by comparing the four migration cases to matched VAD controls.

\section{Material and Methods}

The VADs were inserted, by nine different experienced (at least 20 ports inserted) anesthesiologists, as an outpatient procedure using the percutaneous technique with a Seldinger wire and peal-off introducer. Catheters were inserted in the subclavian vein using a lateral approach. Arrow VADs (A port and low profile models) with preattached silicone catheter (9.6 French, Arrow international vascular access systems, Reading, PA, USA) and Bardports (infusional and low profile) VAD with attachable polyurethane catheters (9.6 French; Bard access systems, Salt Lake City, UT, USA) were used. The procedure was performed with fluoroscopic guidance and an immediate postoperative chest $\mathrm{x}$-ray, with the patient sitting, was performed.

The VAD was clinically checked, every 14 days by the oncology nurse according to the principles outlined by Schulmeister [10] and every 28 days by the physician (PÖ) over a period of 24 weeks, and special emphais was put on prespective evaluation of tolerability i.e. VAD complications, the primary endpoint of the study [19]. A chest $\mathrm{x}$-ray was performed if malfunction of the VAD was suspected (difficulties to aspirate, symptoms on the neck, slow in- fusion rate, infection etc occurred). VADs were in place for 8 months unless metastatic disease was encountered.

Four cases with symptomatic catheter migration were identified from November 1997 to July 2001 at the Department of Oncology at Helsinki University Central
Hospital. Three patients were among the 88 VAD patients with colorectal cancer. The fourth case, with osteosarcoma, was receiving multiplex chemotherapy via VAD. The three cases and 12 controls were included in the prospective randomized LIPSYT study

(ISRCTN98405441). The Ethical Review Board at Helsinki University Hospital approved the protocol and a written informed consent was obtained from all patients.

The four cases of catheter migration were compared with 12 controls, selected from the same VAD population of 88 patients. The 12 controls were matched for sex (male), age ( $+/-5$ years), body mass index (BMI $+/-1)$, physical activity level (occupationally and in leisure time: high, moderate, low), and implantation site (right subclavian vein). A radiologic measurement technique for assessing catheter tip position was modified from Nazarian et al. [20]. Position 1 was allocated when the catheter tip was positioned at the junction of innominate veins with the superior vena cava. Positions 2 - 6 were allocated when the tip was positioned at the uppermost, upper, middle, lower, and lowermost superior vena cava, respectively. Position 7 superior vena cava and right atrial junction, and position 8 when in the right atrium (Figure 1). All chest x-rays were checked by one radiologist (SB). The Filemaker database search modality was used to process the raw data, with sex, age, BMI added stepwise into the model and physical activity level was added as the last criterion. When the best control had been chosen right subclavian insertion site was verified. Nonparametric Mann-Whitney-U test was used for comparison between the cases and controls. All statistical significance tests were bilateral at the 5\% level. StatView statistics (SAS institute, Abacus concepts incorporation, Berkeley, CA, USA) were used.

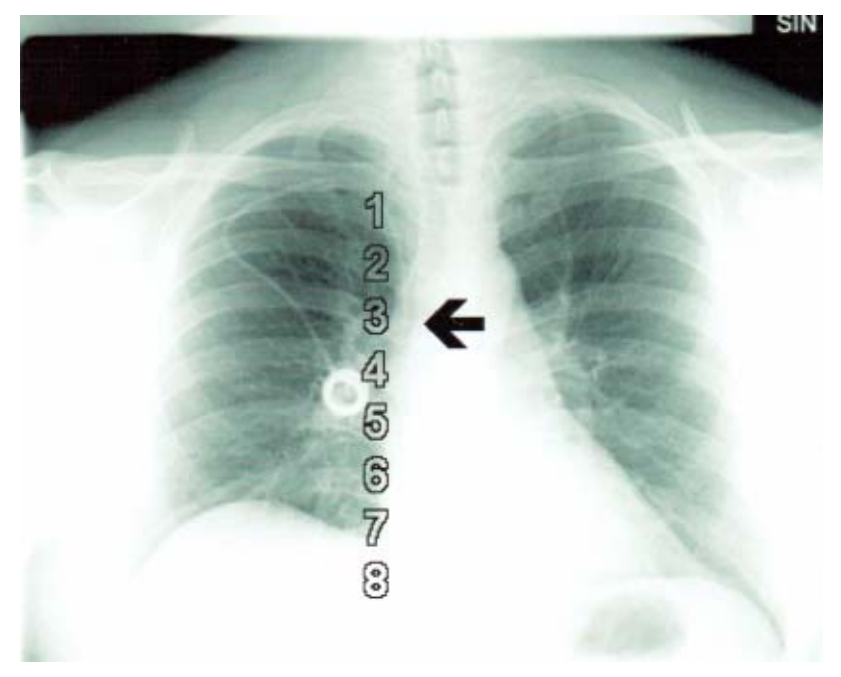

Figure 1. Chest radiograph in case 4 after VAD insertion. The position was 3 (arrow) in catheter tip grading from 1 8 on chest $x$-ray with patient sitting. 


\section{Results}

Case 1. $57-y r$ male $(178 \mathrm{~cm} / 80 \mathrm{~kg} / \mathrm{BMI} 25$, right-handed) with rectosigmoid carcinoma had an Arrow A port. He noted swelling and pain for 5 days in his neck two months after insertion. A catheter migration into the internal jugular vein was noted on chest x-ray and he had an elevated C-reactive protein level $(30 \mathrm{mg} / \mathrm{l})$, leucocytosis $\left(14.4 \times 10^{12} / 1\right)$ and hyper sedimentation $(18 \mathrm{~mm} / \mathrm{h})$. At the time symptoms started he had been decorating the ceiling with a heavy paintbrush.

Case 2. 55-year male, (170 cm/92 kg/BMI 32, righthanded) with rectal cancer had an Arrow A Port. After adjuvant chemotherapy he had elevated CEA and liver metastases were observed. The catheter was seen in the internal jugular vein on chest x-ray performed due to planned liver resection at 12 months from VAD insertion. On enquiry the patient reported constant discomfort in the neck after heavy gym workout 15 days earlier.

Case 3. 63-yr male, (184 cm/96 kg/BMI 28, righthanded) with rectal carcinoma had a small Bardport. Pain and swelling on the neck was noted 2 months after insertion and catheter migration was visualized on chest $\mathrm{x}$-ray. Pain had started 7 days earlier after upper limb extensions at lifting heavy things on a high shelf.

Case 4. 36-yr male, (170 cm/90 kg/BMI 31, righthanded), with an osteosarcoma, had a normal Bardport inserted (Figure 1). Five months later catheter migration was noted on a chest x-ray (Figure 2) and the patient had experienced discomfort in his neck for 10 days starting after weight lifting.

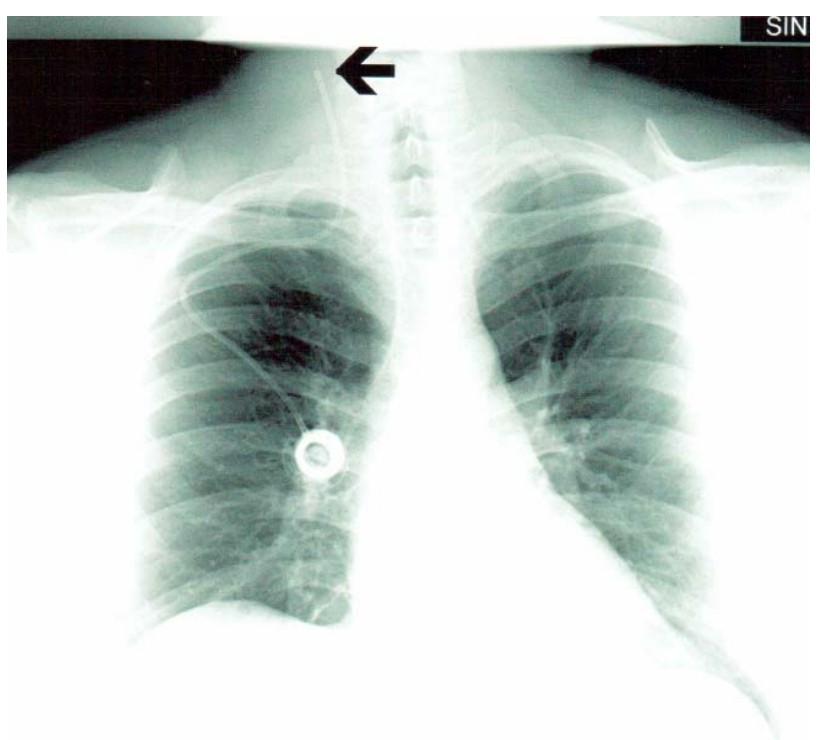

Figure 2. Chest radiograph in case 4 , with catheter migration into the internal jugularis vein (arrow) after weight lifting.

\section{Comparison of Cases and Controls}

When comparing the four cases with the twelve controls: the type of port, catheter material, catheter length, complications at insertion and at the use, and the physical activity level were similar (Table 1).

When assessing catheter tip position with numbering from 1 to 8 (Figure 1) the cases had a median position 3 (range 3 - 4) and controls 6 (range 4 - 8) ( $\mathrm{P}=0.0044)$. Median difference was 3 and $95 \%$ confidence interval was $1-5$.

\section{Discussion}

Vascular access devices are of utmost value in chemotherapy administration. Continuous infusion 5-fluorouracil dosing is the mainstay of colorectal cancer chemotherapy both in the adjuvant and in the metastatic setting. Peripheral catheterization problems are increasingly the reason for VAD insertion, as palliative chemotherapy becomes more efficient and treatment times prolong. To our knowledge prospective assessment of VAD safety in a randomized adjuvant study setting has not been done, though the clinical implications of VAD safety are of utmost importance. Mechanisms for prevention of potentially serious VAD complications are warranted.

VADs are thus the cornerstone in medical oncology, but linked with complications of which catheter migration is potentially hazardous. Symptomatic catheter migration has been reported in $0 \%-3.5 \%$ of patients, and has usually been considered a minor complication which results in catheter malfunction and patient discomfort [11-15]. The $3 / 88(3.4 \%)$ in our randomized material is among the highest noted.

Table 1. Patient characteristics.

\begin{tabular}{ccc}
\hline & $\begin{array}{c}\text { Cases } \\
\mathrm{n}=4 \text { males }\end{array}$ & $\begin{array}{c}\text { Controls } \\
\mathrm{n}=12 \text { males }\end{array}$ \\
\hline $\begin{array}{c}\text { Age (yrs): } \\
\text { median (range) }\end{array}$ & $56(36-63)$ & $56(32-68)$ \\
BMI $\left(\mathrm{kg} / \mathrm{m}^{2}\right):$ \\
median (range) \\
$\begin{array}{c}\text { Physical activity level: } \\
\text { high/moderate/low } \\
\text { Port model: } \\
\text { A port/Bard Port }\end{array}$ & $29(25-32)$ & $28(24-33)$ \\
$\begin{array}{c}\text { Catheter material: } \\
\text { silicone/polyurethane } \\
\text { Complications: } \\
\text { insertion/use }\end{array}$ & $2 / 1 / 1$ & $6 / 2 / 4$ \\
$\begin{array}{c}\text { Catheter length (cm): } \\
\text { median (range) }\end{array}$ & $2 / 2$ & $8 / 4$ \\
$\begin{array}{c}\text { Catheter tip position 1 - 8: } \\
\text { median (range) }\end{array}$ & no/4 migrat. & no/no \\
\hline
\end{tabular}


Catheter tips positioned at lowest third of the vena cava are considered optimal [21]. Some prefer even lower positioning not more than $4 \mathrm{~cm}$ above the caval-atrial junction as higher positions are related to higher complication frequency in tunneled central venous catheters [22]. Fewest complications have been seen in patients with low superior caval vein or right atrial catheter tips [23]. Conventionally catheter tip positioning to the low superior vena cava has been recommended due to the risk of cardiac perforation with the catheter tip in the right atrium [21]. However, earlier studies show that essentially all cardiac perforations occur with polyethylene or other rigid catheters inserted [22] and rarely with softer materials. In large patient materials the catheter tip position within the right atrium was considered satisfactory $[14,24]$ and the low tip position was linked to fewer complications [14,22]. A known problem is the catheter tip moving upwards after insertion in Trendelenburg position when resuming upright position [20,25]. A catheter movement occurred in 49 of 50 patients (average, $3.2 \mathrm{~cm}$ $+/-1.8)$ when compared at chest $\mathrm{x}$-rays supine and upright position [25]. It is recommended that the catheter tip should be initially positioned approximately $3-4 \mathrm{~cm}$ more caudally than the desired final position $[20,25]$. In a recent patient series $9.0 \%$ of catheter tips were positioned higher than intended at treatment initiation [26]. In our four migration cases the catheter tip was positioned too high in the superior caval vein.

The mechanism for migration is not known. In the current literature in one patient a sequence starting with herniation of a short segment of the flexible shaft of the catheter into the jugular vein 12 months after insertion and then continuing to a full catheter reversal with thrombosis in the jugularis vein at 15 months was described [27]. This indicates that the process is not instantaneous. The mechanism could be herniation of the catheter, with migration into the jugular vein at sudden increases in intrathoracic pressure as during the Valsalva maneuvers. In all our four cases the Valsalva maneuvers coincided with strenuous upper limb activity at the time of migration, 5 to 15 days before the condition was detected at patient check-ups. Weightlifting or painting the ceiling causes a pumping movement in the subclavian vein, which in combination with increased intrathoracic pressure during strenuous physical activity might start the catheter migration. Vivid vomiting with sudden changes in intrathoracic pressure has also been suggested a mechanism of migration [28]. A shorter catheter predisposes the catheter migration under these conditions. There are likely to be more short catheters among our patients, but in these four right-handed cases with VAD inserted via right subclavian vein, the migration might have occurred due to the simultaneous combination of Valsalva ma- neuvers and pumping movement of the upper limbs or just the right upper limb. The patients' venous morphology, which cannot be assessed on a routine chest $\mathrm{x}$-ray, could predispose to this condition.

Based on these four cases the standard procedure in VAD insertion has been changed in our hospital: catheter length has been adjusted accordingly and catheter tip has to be in the right atrium at insertion. Also the Valsalva maneuvers combined with strenuous upper extremity activity have been prohibited. As a result of these procedures, thereafter only one catheter migration has occurred in approximately 500 inserted VADs; $0.5 \%$. Thus, avoidance of Valsalva maneuvers and heavy upper extremity activity is probably one way to diminish catheter migration in patients even with a proper catheter tip position.

In conclusion catheter migration to the neck is a potentially serious risk with cardiovascular, neurologic and infectious sequels. In clinical practice this risk can be minimized with careful consideration of the catheter tip at insertion for a post procedure position in the low vena cava or upper right atrium. Chest x-ray check-ups for migration are recommended, especially if there are symptoms in the neck. The patients with an implanted venous access system must avoid strenuous upper limb activity with Valsalva maneuvers as this may be the mechanism for catheter migration.

\section{REFERENCES}

[1] D. M. Parkin, F. Bray, J. Ferlay and P. Pisani, "Global Cancer Statistics, 2002," CA: A Cancer Journal for Clinicians, Vol. 55, No. 2, 2005, pp. 74-108. doi:10.3322/canjclin.55.2.74

[2] A. de Gramont, A. Figer, M. Seymour, et al., "Leucovorin and Fluorouracil with or without Oxaliplatin as First-Line Treatment in Advanced Colorectal Cancer," Journal of Clinical Oncology, Vol. 18, No. 16, 2000, pp. 2938-2947.

[3] J. Y. Douillard, D. Cunningham, A. D. Roth, et al., "Irinotecan Combined with Fluorouracil Compared with Fluorouracil Alone as First-Line Treatment for Metastatic Colorectal Cancer: A Multicentre Randomised Trial," Lancet, Vol. 355, No. 9209, 2000, pp. 1041-1047. doi:10.1016/S0140-6736(00)02034-1

[4] H. Kock, M. Pietsch, U. Krause, H. Wilke and F. Eigler, "Implantable Vascular Access Systems: Experience in 1500 Patients with Totally Implanted Central Venous Port Systems," World Journal of Surgery, Vol. 22, No. 1, 1998, pp. 12-16. doi:10.1007/s002689900342

[5] J. M. Davies and R. M. Goldberg, "Treatment of Metastatic Colorectal Cancer," Seminars in Oncology, Vol. 38, No. 4, 2011, pp. 552-560. doi:10.1053/j.seminoncol.2011.05.009

[6] K. M. Tveit, T. Guren, B. Glimelius, et al., "Phase IIII 
Trial of Cetuximab with Continuous or Intermittent Fluorouracil, Leucovorin, and Oxaliplatin (Nordic Flox) versus Flox Alone in First-Line Treatment of Metastatic Colorectal Cancer: The Nordic-Vii Study," Journal of Clinical Oncology, Vol. 30, No. 15, 2012, pp. 1755-1762. doi:10.1200/JCO.2011.38.0915

[7] T. S. Maughan, R. A. Adams, C. G. Smith, et al., "Addition of Cetuximab to Oxaliplatin-Based First-Line Combination Chemotherapy for Treatment of Advanced Colorectal Cancer: Results of the Randomised Phase 3 Mrc Coin Trial," Lancet, Vol. 377, No. 9783, 2011, pp. 21032114. doi:10.1016/S0140-6736(11)60613-2

[8] J. Y. Douillard, S. Siena, J. Cassidy, et al., "Randomized, Phase III Trial of Panitumumab with Infusional Fluorouracil, Leucovorin, and Oxaliplatin (Folfox4) versus Folfox4 Alone as First-Line Treatment in Patients with Previously Untreated Metastatic Colorectal Cancer: The Prime Study," Journal of Clinical Oncology, Vol. 28, No. 31, 2010, pp. 4697-4705. doi:10.1200/JCO.2009.27.4860

[9] E. Van Cutsem, C. H. Kohne, E. Hitre, et al., "Cetuximab and Chemotherapy as Initial Treatment for Metastatic Colorectal Cancer," The New England Journal of Medicine, Vol. 360, No. 14, 2009, pp. 1408-1417. doi:10.1056/NEJMoa0805019

[10] L. Schulmeister, "Management of Non-Infectious Central Venous Access Device Complications," Seminars in Oncology Nursing, Vol. 26, No. 2, 2010, pp. 132-141. doi:10.1016/j.sonen.2010.02.003

[11] M. Lorenz, C. Hottenrott, R. M. Seufert and A. Encke, "A Totally Implantable Permanent Central Venous Access, Long-Term Experience with Subcutaneous Infusion Chambers (German)," Langenbeck's Archives of Surgery, Vol. 373, No. 5, 1988, pp. 302-309. doi:10.1007/BF01276546

[12] R. Poorter, F. Lauw, W. Bemelman, et al., "Complications of an Implantable Venous Access Device (Porta-Cath) during Intermittent Continuous Infusion of Chemotherapy," European Journal of Cancer, Vol. 13, No. 1996, pp. 2262-2266.

[13] B. Yung, I. A. Cambell, J. S. Elborn, J. S. Harvey and D. J. Shale, "Totally Implantable Venous Access Devices in Adult Patients with Cystic Fibrosis," Respiratory Medicine, Vol. 90, 1996, pp. 353-356.

[14] R. Schwarz, J. Groeger and D. Coit, "Subcutaneously Implanted Central Venous Access Devices in Cancer Patients: A Prospective Analysis," Cancer, Vol. 79, No. 8, 1997, pp. 1635-1640. doi:10.1002/(SICI)1097-0142(19970415)79:8<1635::AID -CNCR30>3.0.CO;2-X

[15] L. Laurenzi, C. Fimiani, S. Natoli, et al., "Complications with Fully Umplantable Venous Access Systems in Oncologic Patients," Tumori, Vol. 82, 1996, pp. 232-236.

[16] M. Hollyoak, T. Ong and J. Leditschke, "Critical Appraisal of Surgical Venous Access in Children," Pediatric Surgery International, Vol. 12, No. 2-3, 1997, pp. 177182. doi:10.1007/BF01349993

[17] P. Craft, J. May, A. Dorigo, C. Hoy and A. Plant, "Hick- man Catheters: Left-Sided Insertion, Male Gender, and Obesity Are Associated with an Increased Risk of Complications," Australian and New Zealand Journal of Medicine, Vol. 26, No. 1, 1996, pp. 33-39. doi:10.1111/j.1445-5994.1996.tb02904.x

[18] P. Carde, M. Cosset-Delaigue, A. Laplanche and I. Chareau, "Classical External Indwelling Central Venous Catheter versus Totally Implanted Venous Access Systems for Chemotherapy Administration: A Randomized Trial in 100 Patients with Solid Tumors," European Journal of Cancer and Clinical Oncology, Vol. 25, No. 6, 1989, pp. 939-944. doi:10.1016/0277-5379(89)90151-X

[19] P. Osterlund, T. Ruotsalainen, R. Korpela, et al., "Lactobacillus Supplementation for Diarrhoea Related to Chemotherapy of Colorectal Cancer: A Randomised Study," British Journal of Cancer, Vol. 97, No. 8, 2007, pp. 10281034. doi:10.1038/sj.bjc.6603990

[20] G. Nazarian, H. Bjarnason, C. Dietz, Jr., C. Bernadas and D. Hunter, "Changes in Tunneled Catheter Tip Position When a Patient Is Upright," Journal of Vascular and Interventional Radiology, Vol. 8, No. 3, 1997, pp. 437441. doi:10.1016/S1051-0443(97)70585-2

[21] L. Bishop, L. Dougherty, A. Bodenham, et al., "Guidelines on the Insertion and Management of Central Venous Access Devices in Adults," International Journal of Laboratory Hematology, Vol. 29, No. 4, 2007, pp. 261-278. doi:10.1111/j.1751-553X.2007.00931.X

[22] J. Petersen, J. Delaney, M. Brakstad, R. Rowbotham and C. Bagley Jr., "Silicone Venous Access Devices Positioned with Their Tips High in the Superior Vena Cava Are More Likely to Malfunction," The American Journal of Surgery, Vol. 178, No. 1, 1999, pp. 38-41. doi:10.1016/S0002-9610(99)00124-5

[23] T. M. Vesely, "Central Venous Catheter Tip Position: A Continuing Controversy," Journal of Vascular and Interventional Radiology, Vol. 14, No. 5, 2003, pp. 527-534. doi:10.1097/01.RVI.0000071097.76348.72

[24] G. Stanislav, R. Fitzgibbons Jr., R. Bailey Jr., et al., "Reliability of Implantable Central Venous Access Devices in Patients with Cancer," Archives of Surgery, Vol. 122, No. 11, 1987, pp. 1280-1283. doi:10.1001/archsurg.1987.01400230066012

[25] C. Kowalski, J. Kaufman, S. Rivitz, S. Geller and A. Waltman, "Migration of Central Venous Catheters: Implications for Initial Catheter Tip Positioning," Journal of Vascular and Interventional Radiology, Vol. 8, No. 3, 1997, pp. 443-447.

[26] R. DeChicco, D. L. Seidner, C. Brun, et al., "Tip Position of Long-Term Central Venous Access Devices Used for Parenteral Nutrition," Journal of Parenteral and Enteral Nutrition, Vol. 31, No. 5, 2007, pp. 382-387. doi: $10.1177 / 0148607107031005382$

[27] P. Rasuli, D. Hammond and I. Peterkin, "Spontaneous Intrajugular Migration of Long-Term Central Venous Access Catheters," Radiology, Vol. 182, No. 3, 1992, pp. 822-824.

[28] M. Binnebosel, J. Grommes, K. Junge, et al., "Internal Jugular Vein Thrombosis Presenting as a Painful Neck 

Adjuvant Colorectal Cancer Chemotherapy

Mass Due to a Spontaneous Dislocated Subclavian Port Catheter as Long-Term Complication: A Case Report,"
Cases Journal, Vol. 2, No. 7991, 2009, p. 7991. doi:10.4076/1757-1626-2-7991 